\title{
Emphysematous Osteomyelitis - A Rare Cause of Gas in Spine - A Case Report
}

\author{
Mahesh BH, Upendra BN, Vijay S*, Arun Kumar GC and Srinivas Reddy
}

Jain Institute of Spine Care and Research, Bhagwan Mahaveer Jain Hospital, Millers road, Vasanthnagar, Bangalore, Karnataka, India

\begin{abstract}
Emphysematous osteomyelitis is rare condition characterised by the presence of intraosseous gas. About 25 such cases have been reported in the literature with only 10 cases involving spine. These infections are commonly seen in elderly morbid patients and are highly fatal. We report a 65 year old female patient with history of sudden onset of back pain and weakness of both the lower limbs with radiological images revealing the features of emphysematous osteomyelitis of L5 vertebra, which was treated with surgical decompression and stabilisation, and post-operative antibiotics. We also review literature to describe the clinical and radiological features for diagnosis and the treatment options available for such infections.
\end{abstract}

Keywords: Emphysematous osteomyelitis; Gas in spine; Surgical decompression

\section{Introduction}

Emphysematous osteomyelitis is the infection of bone caused by gas forming microorganisms [1]. It's a rare condition with only about 25 cases reported in the literature and only 10 involving spine [2]. These infections are highly fatal with the reported mortality of about $32 \%$. Early diagnosis is essential for initiating appropriate treatment and reducing the mortality. The presence of gas as a sign of infection was first described by Ram et al. in 1981 [3] and is pathagnomic of infection in the appendicular skeleton [1]. However, the diagnosis of emphysematous osteomyelitis involving spine is difficult as the presence of gas within the intradiscal or intraosseous space would more commonly indicate a non-infectious process like degeneration, osteonecrosis or osteitis deformans [1,2]. We describe one such rare case diagnosed early and treated successfully with timely surgical intervention and appropriate post-operative antibiotics. We also review literature and describe clinical and radiological features that would help in differentiating emphysematous osteomyelitis of spine from other non-infectious gas forming conditions.

\section{Case Report}

A 65 year old female patient presented with history of severe low back pain since 12 days with sudden onset of weakness of both lower limbs and inability to walk with bladder retention and bowel involvement since 4 days. Pain was continuous, associated with high grade fever and chills. She was a known hypertensive and a case of rheumatoid arthritis being treated with analgesics and steroids. On examination tenderness was found at lower lumbar spine, bilateral feet were flail with decreased sensations and absent ankle jerk. Per rectal examination revealed decreased perianal sensations with lax anal tone and absent anal wink. Her blood investigations revealed Hemoglobin of 9 , total leukocyte count of $14,000 / \mathrm{cm}^{2}$ of which $86 \%$ were neutrophils. Her blood urea and serum creatinine were $63 \mathrm{mg} /$ $\mathrm{dl}$ and $1.9 \mathrm{mg} / \mathrm{dl}$. MRI and CT scans showed multilevel stenosis with altered signal intensity within the body of L5 and with epidural abcess with gas within the body of L5 vertebra, epidural space and in the psoas muscle (Figure 1).

Based on the clinical, biochemical and radiological features she was diagnosed with L5 infective spondylitis and degenerative scoliosis, L2-L3, L3-L4, L4-L5, L5-S1 Lumbar canal stenosis, bilateral foot drop and cauda equina syndrome with chronic drug induced renal failure. In presence of dense neurological deficits with source of infection from L5 body and MRI showing significant stenosis, surgical decompression, debridement and stabilisation was contemplated. After stabilization of general condition, she underwent L1-ilium Pedicle screw instrumentation, L2, L3, L4, L5 Laminectomy and decompression of dura and bilateral roots, transpedicular biopsy of L5 vertebral body with posteriolateral fusion under Spinal anaesthesia. Post-operative $\mathrm{X}$ images showed satisfactory scoliosis correction, posterolateral bone grafting and laminectomy (Figure 2). Intraoperatively samples from L5 vertebral body were sent for histopathalogical examination which showed granulation tissue with neutrophils, necrotic bone, air bubble, soft tissue necrosis and Grams stain smear showed Gram negative bacilli with inflammatory granulation tissue (Figure 3).

Post operatively her general condition improved, renal parameters, total leucocyte and neutrophilic counts returned to normal. She was mobilized on wheel chair and catherization was continued. Culture grew E-coli and biopsy was reported as non-tuberculous osteomyelitis. She was started on Injection Magnex, Meropenam and clindamycin, based on culture and sensitivity reports. A week later, she developed repeated fever spikes with discharge from the wound, for which wound lavage was done. Post lavage her fever reduced and dressing remained dry and she was discharged parenteral antibiotics were continued for 6 weeks which was changed to oral antibiotics for next weeks. At 6 months follow up her pain had reduced, she was able to walk with support, regained bladder control and her motor power recovered partially in right ankle. Laboratory reports and imaging studies revealed resolution of infection. ESR was $16 \mathrm{~mm} / \mathrm{hr}$ and CRP was negative, CT and MRI scans showed resolustion of gas shadows and epidural abcess (Figure 4). However L5 vertebral body showed increased collapse with caudal migration of left connecting rod for which she is under observation and advised revision if becomes symptomatic.

\section{Discussion}

Intravertebral or intraosseous gas in the spine is termed as vacuum phenomenon and was first described by Magnusson in 1937 [4]. It

*Corresponding author: Vijay S, Spine Surgeon, Jain Institute of Spine Care and Research, Bhagwan Mahaveer Jain Hospital, Millers road, Vasanthnagar, Bangalore, Karnataka, India-560052, Tel: 9886271303; E-mail vijays_sdumc@yahoo.co.in

Received June 10, 2016; Accepted July 13, 2016; Published July 15, 2016

Citation: Mahesh BH, Upendra BN, Vijay S, Arun Kumar GC, Reddy S (2016) Emphysematous Osteomyelitis - A Rare Cause of Gas in Spine - A Case Report. Spine 5: 320. doi:10.4172/2165-7939.1000320

Copyright: ( 2016 Mahesh $\mathrm{BH}$, et al. This is an open-access article distributed under the terms of the Creative Commons Attribution License, which permits unrestricted use, distribution, and reproduction in any medium, provided the original author and source are credited. 

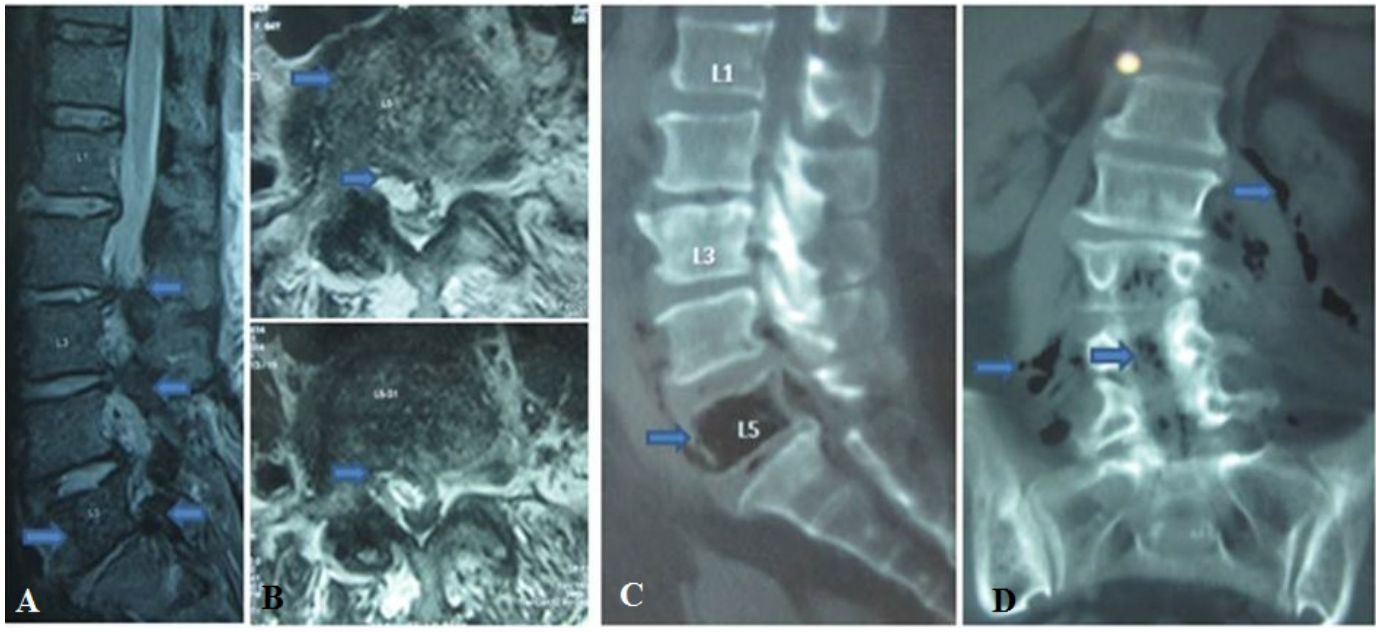

Figure 1: (A and B): T2 weighted mid sagittal and axial at L5 body and L5S1 disc level MRI images of Lumbosacral spine showing multilevel hypertrophied facets and thickened flavum causing significant stenosis at $L 2 L 3, L 3 L 4, L 4 L 5$ and $L 5 S 1$ with altered signal intensity within the body of $L 5$ and epidural abcess with significant dural compression indicated by arrows, with no endplate erosion or collapse of vertebral body and preserved disc height, (C and D): sagittal and coronal CT scan images showing gas within the body of L5 vertebra, epidural space and in the psoas muscle with degenerative scoliosis.
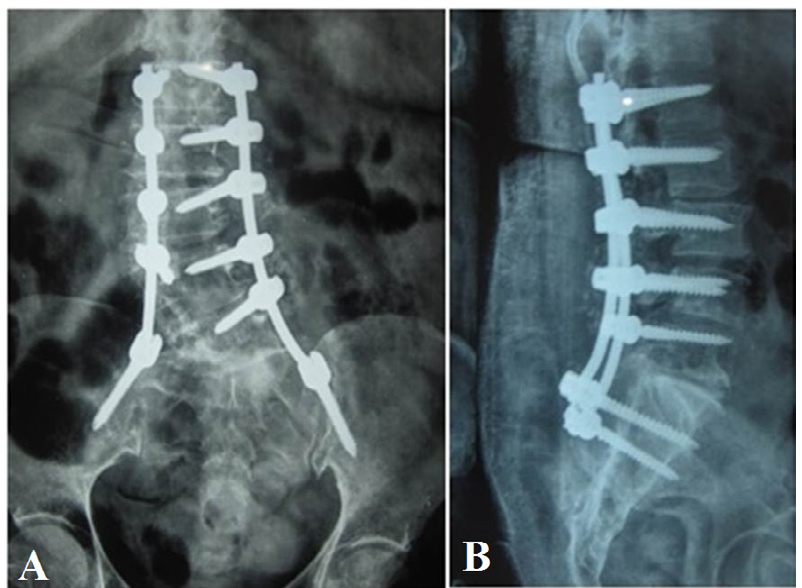

Figure 2: (A and B): Post-operative antero-posterior and lateral $X$ images showing L1- ilium pedicle screw instrumentation with scoliosis correction and posterolateral bone grafting and laminectomy.

can be classified basically into two types as those associated with degenerated disc disease and those with pathological vertebral collapse. Vacuum phenomenon associated with degenerated disc disease was first described in 1942 by Knuttson. It results from the rupture of peripheral fibres of annulus fibrosus with anterior marginal osteophyte preventing the collapse of the disc space and is termed as spondylosis deformans [4]. Sometimes intradiscal gas may extend into the vertebral bodies along with the disc as schmorl's nodule or directly through the defects in the cartilaginous end plates resulting in intravertebral vacuum phenomenon [4]. Degeneration is the most common cause of spinal vacuum phenomenon found in 1-3\% of all spinal radiographic images and in $20 \%$ of the images in elderly [4]. These gas shadows are usually small, localised, surrounded by sclerotic rim and are associated with other degenerative changes without vertebral collapse [4]. Rarely a similar large cyst may be found within the vertebral bodies, commonly in the cervical spine, which are termed as pnuematocysts [5].

Even though majority of the spinal vacuum phenomenon do not warrant any further investigations, not all the cases are benign [4,6]. Feng et al. in their analysis of spinal vacuum phenomenon in pathological vertebral collpase found that traumatic vertebral compression fracture was the most common non degenerative cause, in which intravertebral gas was found in $18.9 \%$ of the cases. While none of the images with spinal metastases showed the vacuum phenomenon, $6.4 \%$ of the collapsed vertebrae with primary neoplasm or multiple myeloma showed gas within the vertebral bodies. Vacuum phenomenon in infections was rare reported in only one of 647 collapsed vertebrae in their series (a prevalence of $0.15 \%$ ) [6].

Luey et al. in their extensive review of case reports of emphysematous ostemyelitis found a total 25 cases reported in the literature [2]. Spine was the most common site to be involved in $40 \%$ of the cases. Most of the patients were elderly with a median age of 51 years and had one or more comorbidities like diabetes and malignancy and one of the patients was on predisolone indicating immunosuppression as a predisposing factor. Fusobacterium and Enterobacteraceae were the common causative organisms. The condition was reported to be highly lethal with fatal course in $32 \%$ of the patients [2].

It is essential to identify vacuum phenomenon due to vertebra osteomyelitis and initiate the treatment early to reduce mortality. 
Citation: Mahesh BH, Upendra BN, Vijay S, Arun Kumar GC, Reddy S (2016) Emphysematous Osteomyelitis - A Rare Cause of Gas in Spine - A Case Report. J Spine 5: 320. doi:10.4172/2165-7939.1000320

A)

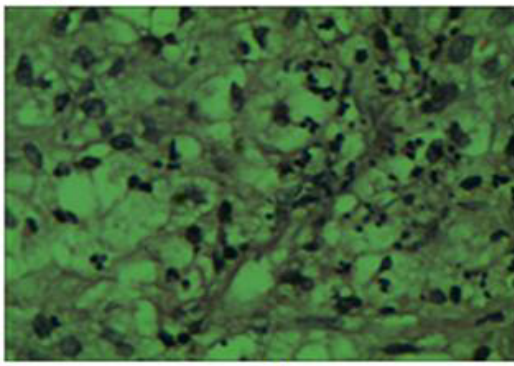

D)
B)

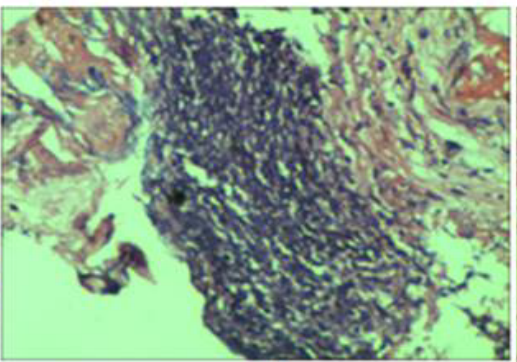

E)
C)

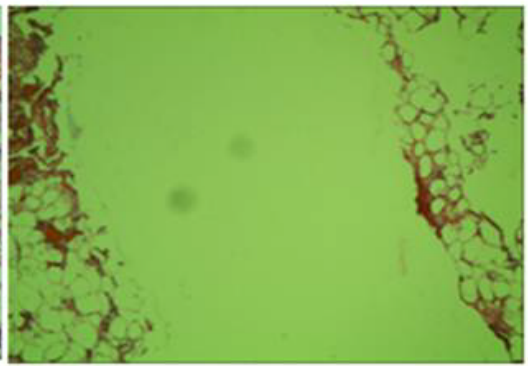

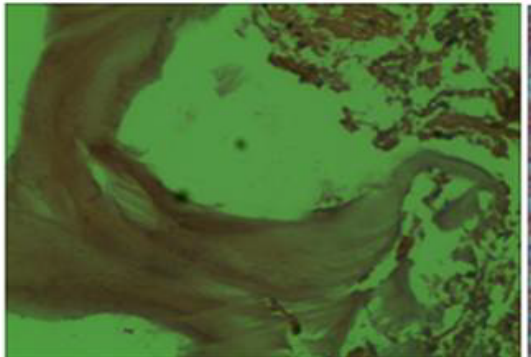

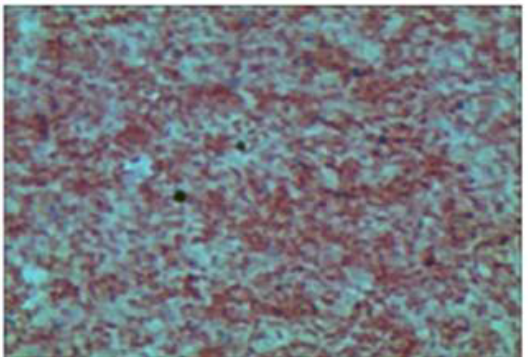

Figure 3: Histopathalogical examination of the biopsy specimen under Hematoxillin-Eosin staining showing (A) Granulation tissue with neutrophils at $400 \mathrm{X}$ (375 microns), (B) Necrotic bone at 200X (750 microns), (C) soft tissue air bubble at 100X (1500 microns), (D) Soft tissue necrosis at $200 \mathrm{X}$ (750 microns) and E) Grams stain smear showing Gram negative bacilli with inflammatory granulation tissue.
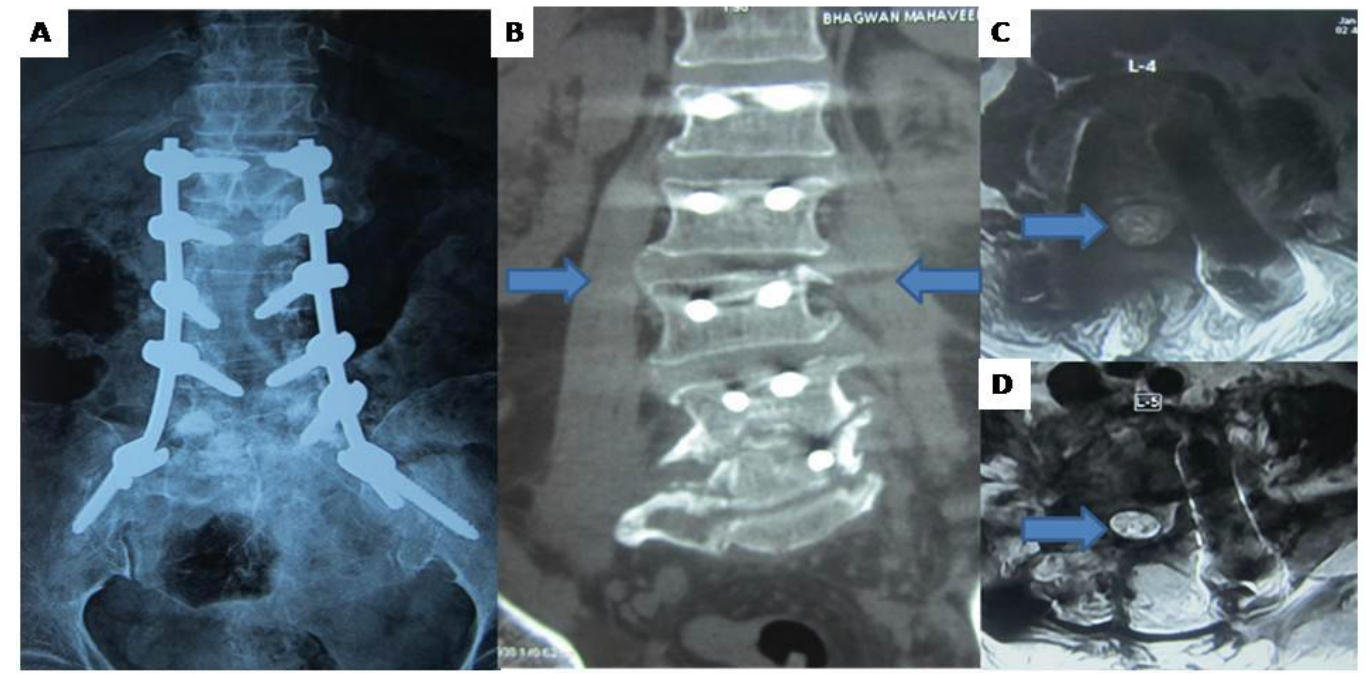

Figure 4: 6 months follow up (A) $X$ ray showing position of implants maintained with posterio-lateral fusion, (B) CT Scan coronal image showing resolution of psoas gas shadow on both sides indicated by arrows and (C) and (D) Axial MRI images at L4 and L5 levels showing resolution of the epidural abcess indicated by arrows.

Differentiating intra vertebral gas shadows due to infection from other commonly found non-infectious conditions is difficult. Feng et al. described some of the radiological features of intravertebral vacuum phenomenon characteristic for different pathological conditions. In vertebral compression fractures and in primary tumors the shape of the gas shadow is usually linear, band like or triangular and is well demarcated. In infective spondylitis the distribution of the gas is usually uneven displaying a bubble like pattern and characteristically extending into the paravertebral soft tissues. A rare case of intravertebral gas secondary to vertebral compression fracture extending into the psoas muscle has also been reported [7].
Our case similar to the description by Luey et al. occurred in elderly with multiple medical comorbidities. Apart from clinical and laboratory features of high grade fever and chills, elevated ESR and leucocyte count the radiological features also helped in the diagnosis of emphysematous osteomyelitis. Presence of intravertebral gas in the absence of vertebral collapse, spreading throughout the vertebral body and extending into the prevertebral tissue and psoas muscle helped in differentiating it from other benign conditions causing vertebral vacuum phenomenon. The diagnosis was confirmed by biopsy and culture. Early surgical decompression and stabilization with appropriate antibiotics helped in the recovery of the patient. 
Citation: Mahesh BH, Upendra BN, Vijay S, Arun Kumar GC, Reddy S (2016) Emphysematous Osteomyelitis - A Rare Cause of Gas in Spine - A Case Report. J Spine 5: 320. doi:10.4172/2165-7939.1000320

\section{Conclusion}

Emphysematous vertebral osteomyletis is a rare cause of gas formation with the vertebral bodies. It should be differentiated from other common benign non infections conditions from clinical, biochemical and radiological features. Early diagnosis, timely surgical intervention and appropriate antibiotics may produce a good outcome.

\section{References}

1. Bielecki DK, Sartoris D, Resnick D, Van Lom K, Fierer J (1986) Intraosseous and Intradiscal gas in association with spinal infection: Report of three cases. AJR Am J Roentgenol 147: 83-86.

2. Luey C, Tooley D, Briggs S (2012) Emphysematous osteomyelitis: A case report and review of the literature. Int J Infect Dis 16: e216-220.
3. Ram PC, Martinez S, Korobkin M, Breiman RS, Gallis HR, et al. (1981) CT detection of intraosseous gas: A new sign of osteomyelitis. AJR Am J Roentgenol 137: 721-723.

4. Coulier B (2004) The spectrum of vacuum phenomenon and gas in spine. JBRBTR 87: 9-16.

5. Tomoaki K, Fujiwara A, Tamai K, Kobayashi N, Saiki K, et al. (2003) Enlarging vertebral body pneumatocysts in the cervical spine. AJR Am J Roentgenol 24: 1707-1710.

6. Feng SW, Chang MC, Wu HT, Yu JK, Wang ST et al. (2011) Are intravertebral vacuum phenomena benign lesions?. Eur Spine J 20: 1341-1348.

7. Van Bockel SR, Mindelzun RE (1987) Gas in the psoas muscle secondary to an intravertebral vacuum cleft: CT characteristics. J Comput Assist Tomogr 11: $913-915$. 